tration was higher in the $\mathrm{P}$ geese (6.44 vs $4.97 \mathrm{~g} / \mathrm{L}$ ). In response to overfeeding, fatty liver resulted from the accumulation of triglycerides $(\approx 95 \%$ of liver lipid content), but also of phospholipids and free and esterified cholesterol. Hepatic storage was greater in the $L$ geese for all lipids except for phospholipids, and resulted in a two-fold heavier fatty liver in this breed ( 1005 vs $485 \mathrm{~g}$ ). Consequently, lipid losses during sterilization, due to cell membrane disruption, were three-fold higher in the $L$ geese ( $26.3 \mathrm{vs} 7.5 \%$ ). A parallel increase in plasma VLDL and HDL concentration (two- to threefold) and in their TG content was noted, especially in the $P$ geese in whom the effect of overfeeding was more pronounced.

In the $P$ goose, the channelling of $T G$ towards secretion, rather than intrahepatic storage, may have been responsible for the lower susceptibility of this breed to liver steatosis. Moreover, in both breeds, overfeeding seemed to induce a relative defect in phospholipid synthesis together with an increase in their secretion, especially as HDL. Consequently, the lack of intrahepatic phospholipids i) may have contributed to prevent cellular hypertrophia and therefore the development of hepatic steatosis and ii) accounted for membrane fragility which was responsible for considerable lipid losses, especially in the $L$ geese.

\section{Measurement of hepatic lipogenesis and cholesterol synthesis with deuterated water $\left(D_{2} O\right)$. F Diraison, C Pachiaudi, M Beylot (Faculté de médecine René-Laën- nec, Inserm Unité 197 and CRNH de Lyon, 69008 Lyon, France)}

Hydrogen atoms are incorporated from body water during fatty acid and cholesterol synthesis. Measurement of deuterium appearance in these molecules during $\mathrm{D}_{2} \mathrm{O}$ administration allows a determination of their rate of synthesis. After validation of this method in the rat, four healthy subjects (normal weight, total triglycerides $0.53 \mathrm{mM}$ ) drank a loading dose of deuterium oxide $(3 \mathrm{~g}$ $\mathrm{D}_{2} \mathrm{O} / \mathrm{kg}$ body water) in two sessions, at 20 and $22 \mathrm{~h}$, then deuterium water enriched at $0.44 \%$ APE ( $6 \mathrm{~g} / \mathrm{kg}$ drinking water) was consumed over the following $60 \mathrm{~h}$ of the study. Deuterium enrichment in plasma water was determined by isotope ratio mass spectrometry (IRMS). Deuterium enrichments in plasma-free cholesterol, palmitate of total triglycerides (TG) and TG of very low density lipoproteins (VLDL) were measured by organic mass spectrometry.

A plateau level in plasma water enrichment was reached at $0.27 \pm 0.01 \%$ APE. Using this value and the deuterium enrichment $\mathrm{mr}$ asured (at $8 \mathrm{~h}$, the third day of the study) in palmitate of TG $(0.8 \pm 0.25 \mathrm{MPE})$, in TG-VLDL $(0.67 \pm 0.24 \mathrm{MPE})$, and in cholesterol $(0.78 \pm 0.18 \mathrm{MPE})$, the calculated fractional synthetic rates of these molecules were, respectively, $13.22 \pm 4.7$ and $10.87 \pm 4.25 \%$ for plasma palmitateTG and palmitate-TG-VLDL and $10.3 \pm$ $1.9 \%$ for plasma-free cholesterol.

These values agree with those reported by Leicht and Jones [(1991) Biol Mass Spectrom 20, 392], who used a delicate technique which involved i) the combustion of TG and cholesterol molecules, and ii) the reduction of water produced for isotopic mass spectrometry analysis. Hellerstein et al [(1991) J Clin Invest 87, 1841], using ${ }^{13} \mathrm{C}$ acetate, obtained lower results; however, there is evidence that labeling heterogeneity of cytosolic acetyl-CoA occurs, the precursor pool in this case [Zhang et al (1994) J Biol Chem 269, 11025].

In conclusion, deuterated water was determined to be a safe and convenient method for measuring the fractional synthetic rates of fatty acids and cholesterol in human beings. 